\title{
Correlation of the expression of YY1 and Fas cell surface death receptor with apoptosis of peripheral blood mononuclear cells, and the development of multiple organ dysfunction in children with sepsis
}

\author{
JUDITH RESÉNDIZ-MARTÍNEZ ${ }^{1}$, JUAN ASBUN-BOJALIL ${ }^{1}$, SARA HUERTA-YEPEZ ${ }^{2}$ and MARIO VEGA ${ }^{3}$ \\ ${ }^{1}$ Servicio de Terapia Intensiva Pediátrica, Hospital General Dr Gaudencio González Garza, \\ Centro Medico La Raza IMSS, 02990 Mexico City; ${ }^{2}$ Unidad de Investigación en Enfermedades Oncológicas, \\ Hospital Infantil de México Federico Gómez S.S.A; ${ }^{3}$ Oncology Research Unit, \\ Oncology Hospital, Siglo XXI National Medical Center, IMSS, 06720 Mexico City, Mexico
}

Received October 5, 2016; Accepted December 7, 2016

DOI: $10.3892 / \mathrm{mmr} .2017 .6310$

\begin{abstract}
Multiple organ dysfunction (MOD) is a lethal complication in children with sepsis. Apoptosis of several cell types is involved in this process, and it is associated with increased Fas cell surface death receptor (Fas) expression. As YY1 transcription factor (YY1) negatively regulates the expression of Fas in cancer models, and is associated with the clinical outcome, it may be important in MOD. The present study aimed to determine the association between the expression of Fas, YY1 and apoptosis in children with sepsis, and its association with MOD, these factors were analyzed in 30 pediatric patients that had been diagnosed with sepsis. Peripheral blood mononuclear cells were purified from patients, and YY1 and Fas protein expression was assessed by immunocytochemistry. Apoptosis was determined by terminal deoxynucleotidyl transferase dUTP nick-end labeling. Sepsis was monitored using clinical parameters, pediatric logistic organ dysfunction (PELOD) score and the pediatric mortality index. The results demonstrated that Fas expression was directly correlated with apoptosis levels and the expression of YY1 was inversely correlated with apoptosis levels. Patients with high levels of apoptosis exhibited increased disease severity and poor clinical outcome. Notably, the findings of the present study demonstrated that there were higher survival rates in patients with high YY1 expression, compared with those with low YY1 expression. Additionally, patients with MOD exhibited lower proportions of apoptotic cells compared with sepsis patients
\end{abstract}

Correspondence to: Dr Mario Vega, Oncology Research Unit, Oncology Hospital, Siglo XXI National Medical Center, IMSS, 330 Avenue Cuauhtemoc, Doctores, 06720 Mexico City, Mexico E-mail: marioi@unam.mx

Key words: apoptosis, sepsis, YY1 transcription factor, multiple organ dysfunctions, Fas cell surface death receptor, Fas-ligand without MOD. Furthermore, the PELOD score was positively correlated with Fas and inversely correlated with YY1 expression. Finally, high apoptosis and low YY1 expression were prognostic factors associated with poor survival rates. These data suggested that YY1 may be important for apoptosis induction via the regulation of Fas during sepsis. Therefore, Fas may be a potential therapeutic target to prevent MOD through regulation of YY1 expression. Furthermore, YY1 and Fas expression in PBMCs may be used to as prognostic markers.

\section{Introduction}

Sepsis is defined as a systemic inflammatory response syndrome caused by infection, which induces an exacerbated inflammatory response of the host immune system, mediated by cytokine-induced immune cells, and causes changes in cellular processes, including apoptosis (1). Globally, severe sepsis, septic shock and the resulting organ failure are the most common causes of mortality in intensive care units (ICUs), with mortality rates of $40-70 \%$. Sepsis is a major cause of mortality, estimated to cause $\sim 250,000$ mortalities in the USA, annually. The survival rates of patients with sepsis and infection in ICUs are poor. In Mexico, a study of 21 hospitals, which included 1,039 children, reported an incidence of sepsis in $16 \%$ of patients, and the most vulnerable age group was 1-5 years (2). In addition, another study conducted in 135 ICUs in Mexico reported that sepsis was one of the most common causes of admission in $27.3 \%$ of ICUs, with pneumonia being the primary cause of sepsis in the majority (33\%) of cases (3). Septic shock was the leading cause of mortality in $30.4 \%$ of ICUs analyzed in the study. In an ICU from Mexico City, sepsis and septic shock were the initial diagnoses on admission $(3,4)$.

Currently, it is not clear whether necrosis or apoptosis have a predominant role in severe sepsis (4). The pathogenesis of multiple organ dysfunction (MOD) and failure in patients with severe sepsis is multifactorial. Sepsis triggers the secretion of pro-inflammatory cytokines, activation of lymphocytes 
and other processes. This syndrome has also been reported to cause immunosuppression by decreasing the number of $\mathrm{T}$ and B lymphocytes as result of an increase in apoptosis (5). A previous study has demonstrated that apoptosis exacerbates sepsis, and elevated expression of $\mathrm{Bcl}-2$ apoptosis regulator (Bcl-2) or administration of caspase inhibitors protects against lymphocyte apoptosis and improves survival in a mouse model of sepsis (6). Sepsis triggers apoptosis in various organs leading to septic shock and MOD. It was previously observed that the failure of these organs was associated with increased expression of Fas cell surface death receptor (Fas), therefore, apoptosis may be correlated with the severity of sepsis (7). During sepsis, the immune system is exposed to bacterial antigens, such as lipopolysaccharide (LPS), which induces tissue damage. Thus, activated immune cell death is a potential mechanism for the induction of $\mathrm{T}$ cell apoptosis and the loss of immune function during sepsis. LPS initiates a rapid immune response and leads to leukocyte activation, coupled with the overproduction of pro-inflammatory mediators, including tumor necrosis factor (TNF)- $\alpha$, interleukin (IL)-1, IL-6, IL-12, interferon- $\gamma$, eicosanoids and free radicals of nitric oxide, which are responsible for tissue damage that leads to MOD. Subsequently, cytokine production switches from pro-inflammatory to anti-inflammatory $\mathrm{Th} 2$ cytokines, including IL-4, IL-5 and IL-10, and monocyte and dendritic cell apoptosis is deactivated, which results in immunosuppression (8-10). Apoptosis affects subpopulations of lymphocytes, including $\mathrm{CD}^{+}$and $\mathrm{CD} 4^{+} \mathrm{T}$ cells, $\mathrm{B}$ cells and dendritic cells. Furthermore, it has been observed that downregulation of the immune response by regulatory $\mathrm{T}$ cells, and deactivation of the immune response mediated by Th1 cells, contributes to immunosuppression in sepsis $(11,12)$. Certain clinical and experimental observations have suggested that apoptotic cell death has an important role in the pathogenesis and development of MOD in patients with sepsis (4). The high mortality observed in patients with severe sepsis and those exhibiting a systemic response to infection may be caused by different levels of apoptosis. Recent research concluded that TNF- $\alpha$ and Fas-ligand (FasL) may participate in the induction of apoptosis during severe sepsis (8). Fas expression has been previously reported to be associated with decreased inflammation and MOD, and increased expression of Fas-FasL was reported to be associated with liver dysfunction, disseminated intravascular coagulation and the development MOD in children with severe sepsis (8). The mechanisms of organ failure caused by sepsis remain unclear, but may be associated with cell death, indicating that cellular function is impaired. Organ damage during sepsis varies from marked and accelerated apoptosis in intestinal epithelium and splenic dendritic cells, to minimal levels of cell death in other organs (4). In studies in rats, macrophage apoptosis was increased following the induction of sepsis, similar to the effect on monocytes (13). A previous study on patients postmortem have indicated that the degree of apoptosis in lymphocytes is associated with the severity of sepsis (14).

The YY1 transcription factor (YY1), also termed $\delta$, Nuclear Factor Erythroid 1 (NF-E1) or ubiquitous transcription factor belonging to the GLI-Kruppel class of zinc finger proteins (UCRBP), is a multifunctional ubiquitous transcription factor with four zinc finger domains and two specific functions; to act as a transcriptional repressor or activator. YY1 has been implicated in various normal biological processes, including embryogenesis, differentiation, cell replication and proliferation. It is reported that YY1 binds to the silencing promoter region of Fas, which decreases the expression of this death receptor, resulting in resistance to apoptosis mediated by Fas-FasL. Thus, inhibition of YY1 activity increases in Fas expression and sensitizes tumor cells to Fas-mediated apoptosis (15-17). MOD is a lethal complication in children with sepsis, and apoptosis has an important role in its pathogenesis. Certain studies have suggested an association between apoptosis and sepsis severity in children (18-20) and the mechanisms involved may include the Fas-FasL system. YY1 regulates the expression of Fas and, therefore, mediates apoptosis via Fas-FasL in tumor cells. However, at present, to the best of our knowledge, no studies have demonstrated the role of YY1 in the pathogenesis of sepsis. The present study assessed the correlation of Fas and YY1 expression with apoptosis in peripheral blood cells, and with MOD in children with sepsis.

\section{Materials and methods}

Patient information. The present study included a cohort of patients $(n=30)$ aged between 3 months and 16-years-old. All were diagnosed with sepsis and admitted to the pediatric ICU of the General Hospital from Medical Center La Raza, IMSS (Mexico City, Mexico). The present study was approved by the National Commission for Scientific Research and the Ethics Board of the Mexican Institute for Social Security (IMSS R-2008-3502-13) and was performed in accordance with the Declaration of Helsinki (21). A written informed consent was obtained from each patient and all samples were obtained according to National Commission for Scientific Research of the IMSS (CIS) guidelines. The inclusion criteria were set and the following clinical information was collected: Name; registration card no.; sex; age; initial diagnosis and previous antimicrobial treatments. All patients in the cohort were under 16-years-old, they were admitted with different diagnoses and were diagnosed with sepsis during their stay at the hospital. The sepsis diagnosis was treated as the day 0 time point. A peripheral blood sample was obtained for immunostaining and terminal deoxynucleotidyl transferase dUTP nick-end labeling (TUNEL). Clinical evaluation was performed every $24 \mathrm{~h}$ to monitor sepsis, pediatric logistic organ dysfunction (PELOD) and pediatric mortality index (PIM-2), until multiple organ dysfunction developed or clinical improvement was observed. Integral clinical evaluation was performed, and special attention was paid to disease severity and any additional complications. The severity of the disease was determined using PIM-2 and scoring was performed with the first hour of arriving at the pediatric ICU. The presence of MOD was determined using the PELOD scale. Each patient was evaluated every $24 \mathrm{~h}$ during their ICU stay.

MOD was defined as the presence of dysfunction in two or more organs or systems, including the cardiovascular, respiratory, neurological, hematological, renal, gastrointestinal and hepatic systems. Complete blood counts, urinalysis, polyculture and chest radiographs were performed. If a serologic sample was not available, one was collected in the pediatric ICU. Furthermore, additional studies were performed according to 
severity of the disease or clinical conditions. Blood samples $(5 \mathrm{ml})$ were collected from a peripheral vein in an anticoagulant tube and were sent to the research laboratory for analysis within 30-120 min following sample collection. Apoptosis in peripheral blood cells was measured using TUNEL analysis, and Fas and YY1 expression were determined via immunocytochemistry. A total of 10 peripheral blood samples were taken from patients who attended the clinical laboratory for pre-operative studies for use as control samples. The control patients did not have hematologic disease, infection or cancer, and informed consent was obtained for the use of the samples.

Purification of peripheral blood mononuclear cells (PBMCs). Peripheral blood was diluted 1:1 with saline solution (SS; $0.9 \%$ $\mathrm{NaCl}$ ) and corresponding volume of sterile Lymphoprep ${ }^{\mathrm{TM}}$ (Cosmo Bio USA, Inc., Carlsbad, CA, USA) was used. The mixture was centrifuged at $355 \times \mathrm{g} /$ room temperature for $30 \mathrm{~min}$. Subsequently, the intermediate layer formed was separated, which corresponds to mononuclear cells, and then washed with an equal amount of SS and centrifuged for $20 \mathrm{~min}$ at $355 \mathrm{x} \mathrm{g} / \mathrm{room}$ temperature. Finally, the supernatant was decanted and the pellet was resuspended in $1 \mathrm{ml}$ of SS ready for counting.

Preparation of cells slides using Cytospin. Following purification, PBMCs were counted using trypan blue. After viable cells were counted, the concentration was adjusted to $5 \times 10^{4}$ cells $/ \mu 1$ when preparing cell slides. Each slide had $200 \mu \mathrm{l}$ of cell suspension added and was placed in a Cytospin centrifuge (Cytospin ${ }^{\mathrm{TM}}$ 4; Thermo Fisher Scientific, Inc., Waltham, MA, USA). Subsequently, cells slides were dried at room temperature and were fixed with $3 \%$ formaldehyde at $4^{\circ} \mathrm{C}$ for $20 \mathrm{~min}$ and stored for subsequent immunostaining.

Detection of apoptosis by TUNEL assay. DNA fragmentation was determined by in situ TUNEL assay on cells from patients with sepsis. Briefly, the cell slides were treated with sodium citrate solution $(\mathrm{pH}=6 ; 0.01 \mathrm{M})$ for $20 \mathrm{~min}$ at $90^{\circ} \mathrm{C}$ in a water bath. Activity of endogenous peroxidase was removed with hydrogen peroxide in $3 \%$ methanol for $25 \mathrm{~min}$. The cell slides were blocked with swine normal serum (Sigma-Aldrich; Merck Millipore, Darmstadt, Germany) at $2 \%$ for $2 \mathrm{~h}$ at room temperature. Subsequently, $50 \mu 1$ enzyme terminal deoxynucleotidyl transferase reaction mixture (TUNEL Label mix, Roche Diagnostics, Basel, Switzerland), was added to slides and incubated for $30 \mathrm{~min}$ in a humidity chamber in the dark at $37^{\circ} \mathrm{C}$, slides were washed for 5 times for 5 min with PBS. Anti-fluorescein antibody conjugated horseradish peroxidase (20 $\mu$; ; TUNEL POD Detection system, Roche Diagnostics), was added and incubated at $37^{\circ} \mathrm{C}$ for $30 \mathrm{~min}$ in darkness. Following washing, color development was carried out with the addition of diaminobenzidine (DAB) Under a light microscope at x20 magnification. The samples were counter-stained with hematoxylin, dehydrated and embedded in resin for subsequent analysis under a light microscope. To avoid inter-assay variation, the TUNEL reaction was performed simultaneously on all slides, with the same stock and enzymatic reaction development with the DAB chromogen was performed at the same time. A semi-quantitative assessment of cells staining was conducted by an expert pathologist, who was blinded to the samples group. The stained slides were verified by a second expert to ensure consistency in the scoring. Apoptosis was considered high with $>30 \%$ of cells positive for apoptosis and low with $<30 \%$ of positive cells analyzed for each slide in three different areas. Data are presented as positively stained target cells per 100 cells (range $0-100 \%$ positive), per region in the slide (4 regions per slide).

Immunocytochemistry. The sample slides from Cytospin were analyzed by immunocytochemistry as follows. Briefly, antigen retrieval was performed with sodium citrate $(\mathrm{pH} \mathrm{6.0}$; $0.01 \mathrm{M}$ ) for $20 \mathrm{~min}$ at $90^{\circ} \mathrm{C}$ in a water bath, followed by three 5 min washes with $1 \mathrm{X}$ PBS. Endogenous peroxidase activity was removed with $3 \%$ hydrogen peroxide for two $25 \mathrm{~min}$, and the slides were blocked with swine normal serum at $2 \%$ at room temperature for $2 \mathrm{~h}$. Slides were incubated overnight at room temperature in humidity chambers with rabbit anti-YY1 (1:250; catalog no. sc-281), rabbit anti-Fas (1:250; catalog no. sc-1023) or rabbit anti-FasL (1:100; catalog no. sc-6237; Santa Cruz Biotechnology, Inc., Dallas, TX, USA) antibodies, with their respective isotype controls. After washing, the cell slides were incubated with secondary antibody conjugated to biotin (Universal LSAB kit; Dako; Agilent Technologies, Inc., Santa Clara, CA, USA) for $30 \mathrm{~min}$ in a humid chamber. Then conjugated streptavidin horseradish peroxidase (Universal LSAB kit), was added for $30 \mathrm{~min}$. Finally, development was performed by adding DAB substrate (liquid DAB, Dako; Agilent Technologies, Inc.) and the reaction was stopped in tap water. Slides were stained with hematoxylin, were dehydrated and covered with resin and left to dry at room temperature for further light microscopic analysis (Olympus BX-40; Olympus Corporation, Tokyo, Japan). In order to reduce variations among experiments, the reaction was performed for each marker at the same time in all groups. High YY1 expression was defined as $\geq 10 \%$ of cells positive for $\mathrm{YY} 1$.

Data processing. The database was produced and information was processed using the statistical analysis program GraphPad Prism (GraphPad Software, Inc., La Jolla, CA, USA). Data are presented as the mean \pm standard deviation for the number of separate experiments indicated in each case. One-way analysis of variance followed by Tukey's post hoc test was used to compare variance within and among different groups. When necessary, unpaired Student's t-test was used for comparison between two groups. Spearman's correlation analysis was performed for correlation testing. $\mathrm{P}<0.05$ was considered to indicate a statistically significant difference.

\section{Results}

Patient clinical characteristics. The current study included 30 patients diagnosed with sepsis. Clinical data are presented in Table I. For the purposes of the study, the patients were divided into two groups. Group 1 consisted of patients with sepsis and septic shock $(n=19)$, and group 2 consisted of septic patients with MOD $(n=11)$. The gender distribution was 14 females (47\%) and 16 males (53\%). The average age of group 1 and group 2 patients was 51 months (range, 3-108 months) and 30.7 months (range, 2-180 months), respectively. The PELOD scores were as follows: $0-10$ points in 7 patients 
Table I. Age and characteristics of patients with sepsis.

A, Age of patients with sepsis involved in the study

\begin{tabular}{lc}
\hline Value & Age (months) \\
\hline Range & $3-180$ \\
Mean & 41.44 \\
\hline
\end{tabular}

B, Characteristics of patients with sepsis

\begin{tabular}{lc}
\hline Characteristic & Number of patients (\%) \\
\hline Gender & \\
Female & 47.00 \\
Male & 53.00 \\
Main site of infection/septic origin & \\
Lung & 26.00 \\
Heart & 10.00 \\
Central nervous system & 6.60 \\
Surgical site & \\
Heart & 30.00 \\
Abdomen & 23.30 \\
Cranium & 3.30 \\
Septic stage & \\
Severe sepsis & 33.00 \\
Septic shock & 33.00 \\
Multiple organ dysfunction & 40.00 \\
Outcome & \\
Survival & 66.60 \\
\hline
\end{tabular}

Total $n=30 ; 9$ developed severe sepsis, 10 septic shock and 11 multiple organ dysfunction. Of the 30 patients, 10 did not survive (33.33\%).

(mortality prediction $0.0-20 \%$; no mortalities); $11-20$ points in 9 patients (mortality prediction $1.3-1.7 \%$; no mortalities); 21-30 points in 8 patients (mortality prediction $20.8-32.3 \%$; 5 mortalities); and $>30$ points in 6 patients (mortality prediction $87.7-90 \%$; 5 mortalities). A PIM-2 of $0.80-15.40 \%$ applied to 20 patients and no mortalities were observed in this group, however, a PIM-2 of $>16 \%$ was observed for 10 patients and none of these patients survived. A total of 10 patients in group 2 had PIM-2s $>16 \%$. In group 1, one patient had a PIM-2 of $20 \%$ (data not shown). A total of 17 of 30 patients developed sepsis following surgery (56.6\%), as indicated in the Table I. Afflictions associated with sepsis and MOD are presented in Table II. Sepsis etiology is presented in Table III and blood culture results are presented in Table IV. The mean hospitalization period was 9.5 days with a maximum of 23 days and a minimum of 2 . The total lethality was $33.3 \%$, with $83.3 \%$ in group 2 and $0.0 \%$ in group 1 .

Levels of apoptosis, and the expression levels of Fas and YYI in PBMCs from pediatric patients with sepsis. The present study investigated the levels of apoptosis in PBMCs from children with sepsis using an in situ TUNEL assay. Fig. 1A
Table II. Sepsis etiology in pediatric patients with sepsis that also developed multiple organ dysfunction.

\begin{tabular}{ll} 
Etiological factor & Number of patients \\
\hline Heart post-surgery & 2 \\
Canal atrioventricular correction & 1 \\
Tetralogy of Fallot correction & 1 \\
Anomalous venous connection correction & \\
Post-infection & 3 \\
Nosocomial pneumonia & 1 \\
Abdominals sepsis & 1 \\
Endocarditis & 1 \\
Neuroinfections & 1 \\
Ventriculitis & \\
\hline
\end{tabular}

Table III. Sepsis etiology in pediatric patients without MOD.

Etiological factor Number of patients

\begin{tabular}{lr}
\hline Abdominal sepsis & 1 \\
Laparoscopy appendicitis complicated & 3 \\
Intestinal occlusion post-surgery & 2 \\
Nosocomial pneumonia & 5 \\
Endocarditis & 1 \\
Pericardial effusion from chronic & 1 \\
kidney disease & 1 \\
Craniotomy tumor resection & 2 \\
Heart post-surgery &
\end{tabular}

Table IV. Results of blood culture testing in patients with sepsis involved in the study $(\mathrm{n}=30)$.

Results of blood culture Number of patients

Candida albicans

7

Staphylococcus coagulase negative

Klebsiella

4

Pseudomona

1

H. faecalis

2

Negative

15

presents a representative micrograph of the TUNEL-stained cells in patients with sepsis and healthy controls. The cells from patients with sepsis demonstrate morphological changes with a scant cytoplasm, a fragmented nucleolus (apoptotic bodies) and vacuoles, compared with cells from healthy controls (Fig. 1A). Analysis of the percentage of TUNEL-positive cells was performed as described in the Materials and methods. Fig. 1A (bottom panel) demonstrates a significant increase in the number of apoptotic cells in patients with sepsis compared with the healthy controls 
A Negative control
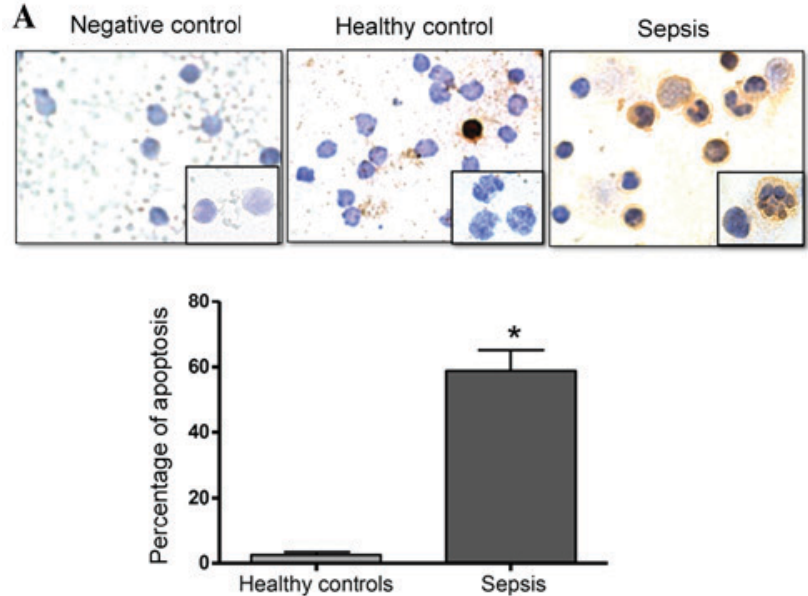

C
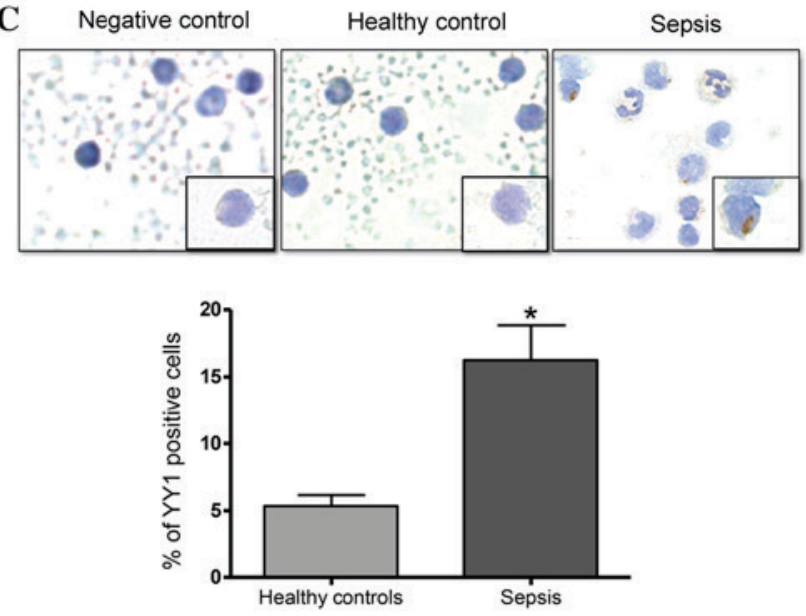

E

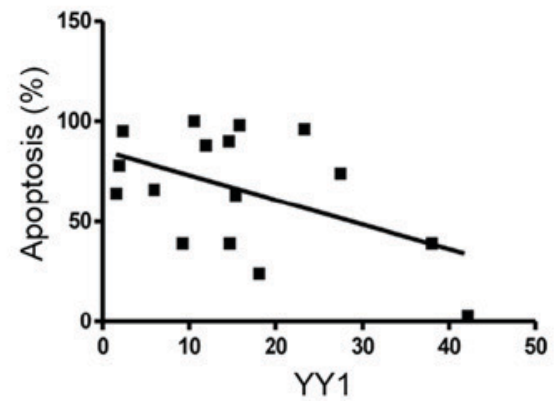

F
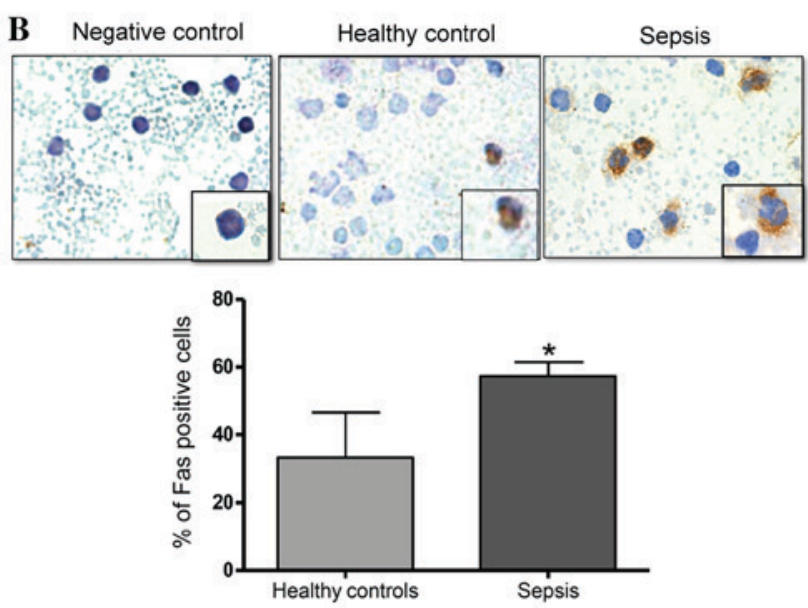

D

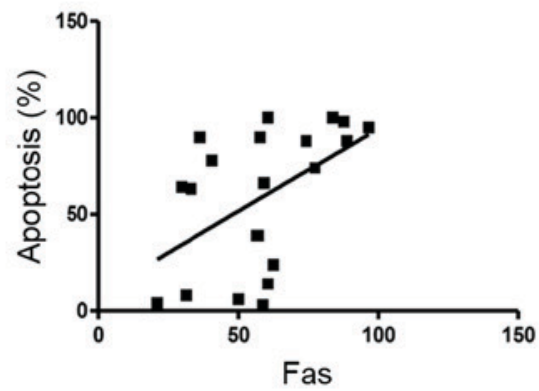

Negative control

Healthy control

Sepsis
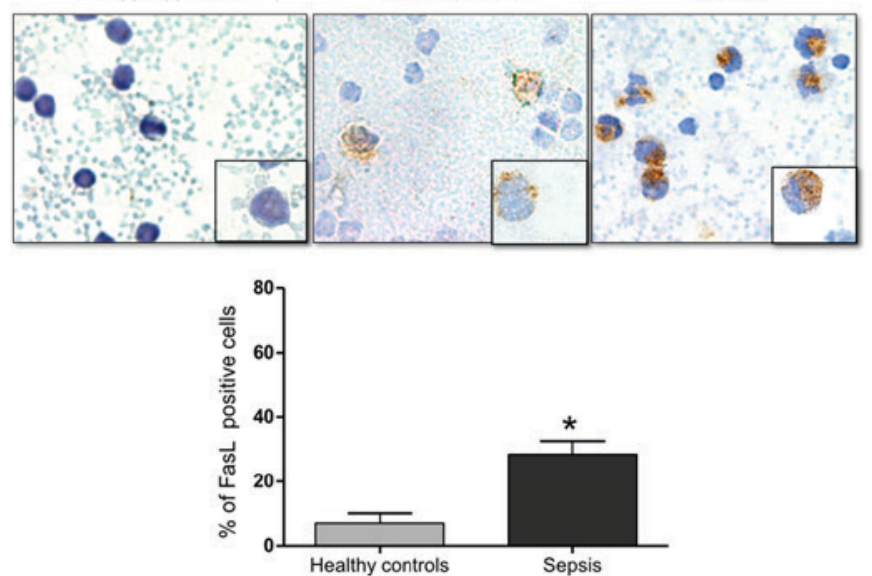

Figure 1. Septic pediatric patients exhibit increased levels of apoptosis. (A) Apoptosis was evaluated using an in situ TUNEL assay in PBMCs from pediatrics patients with sepsis. Upper panels: Negative control assay with no enzyme, healthy control PBMCs and PBMCs from pediatric patients with sepsis. Magnification at x40, and x100 in lower right square. Bottom panel: Quantification of TUNEL-positive cells (\%) in healthy controls and patients with sepsis ( $\mathrm{P}=0.0007$ vs. healthy controls; Student's $t$ test). (B) Fas expression was determined by immunocytochemistry on PBMCs from pediatric patients with sepsis. Upper panels: Isotype control, healthy control and patient with sepsis. Magnification at x40, and x100 in lower right square. Bottom panel: Quantification of Fas-positive cells (\%) in healthy controls and patients with sepsis (" $\mathrm{P}=0.022$ vs. healthy controls; Student's $t$ test). (C) YY1 expression was determined by immunohistochemistry in PBMCs from pediatric patients with sepsis. Upper panels: Isotype control, healthy control and patient with sepsis. Magnification at x40, and x100 in lower right square. Bottom panel: Quantification of YY1-positive cells (\%) in healthy controls and patients with sepsis ("P<0.05 vs. healthy controls; Student's $t$ test). Correlation analysis between apoptosis and (D) Fas and (E) YY1 expression in PBMCs from pediatric patients with sepsis. ("P<0.05; $\mathrm{r}=0.5013$ and 0.4003 , respectively; Spearman's correlation analysis). (F) FasL expression was determined by immunocytochemistry in PBMCs from pediatric patients with sepsis. Upper panel: Isotype control, healthy control and patient with sepsis. Magnification. x40, and x100 on lower right square. Bottom panel: Quantification of FasL-positive cells (\%) in healthy controls and patients with sepsis ( $\mathrm{P}=0.033$ vs. healthy controls; Student's $t$ test). Data are presented as the mean \pm standard deviation of three independent experiments. TUNEL, terminal deoxynucleotidyl-transferase-mediated dUTP nick end labeling; PBMCs, peripheral blood mononuclear cells; Fas, Fas cell surface death receptor; YY1, YY1 transcription factor; FasL, Fas ligand. 
A

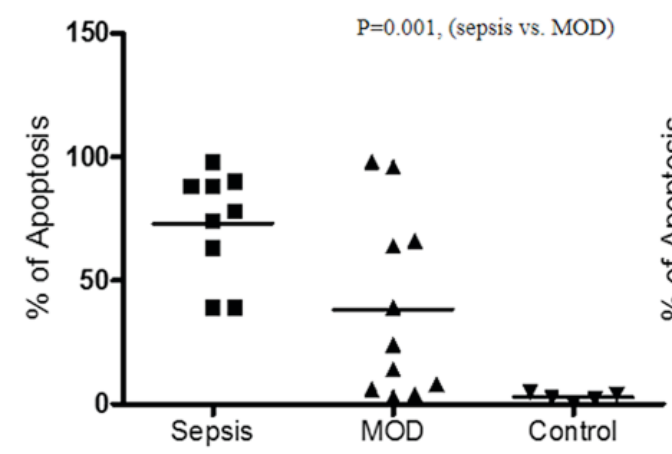

B

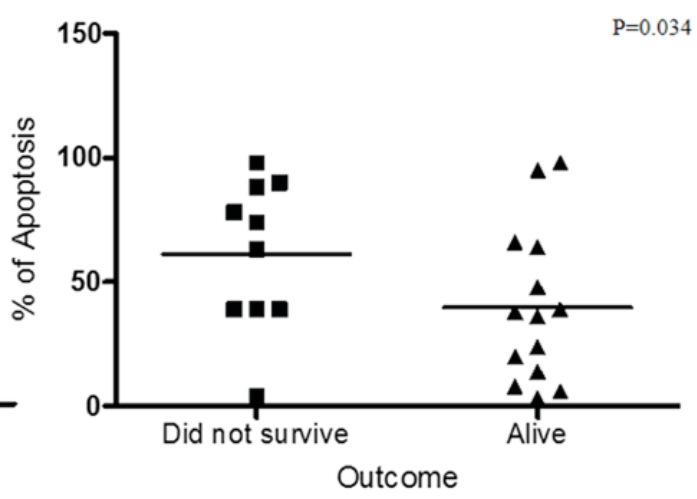

Figure 2. Percentage of TUNEL-positive (apoptotic) cells in PBMCs from pediatric patients with sepsis. (A) Percentage of apoptotic cells was compared between the severities of sepsis. $(\mathrm{P}=0.001$, sepsis vs. MOD). (B) Correlation analysis between levels of apoptosis and outcome of patients with sepsis $(\mathrm{P}=0.0344$; Student's t-test; alive vs. did not survive). TUNEL, terminal deoxynucleotidyl transferase dUTP nick-end labeling; PBMC, peripheral blood mononuclear cell; MOD, multiple organ dysfunction.

$(\mathrm{P}=0.0007)$. Additionally, the expression of Fas in PBMCs from patients with sepsis and healthy controls was analyzed using immunocytochemistry. Fig. 1B presents representative micrographs of Fas expression and demonstrates an increase in Fas expression in patients with sepsis compared with the healthy controls. The expression of these proteins was predominantly observed in the cytoplasm and the membrane. Analysis of the percentage of Fas-positive cells was performed as described in the Materials and methods. Fig. 1B (bottom panel) presents the quantified expression of Fas. Notably, there was a significant increase in the percentage of Fas-positive cells in patients with sepsis compared with in the healthy controls $(\mathrm{P}=0.022)$. Normal $\mathrm{IgG}$ was used as a negative control for immunostaining. Fig. 1C presents representative micrographs of YY1 expression in patients with sepsis and healthy controls, in which a modest increase in YY1 expression was observed in patients with sepsis compared with healthy controls. YY1 expression was predominantly detected in the cytoplasm, however, notably, YY1 expression was also observed in the nucleolus. Additionally, cells from patients with sepsis exhibited a morphology that was characteristic of infection, with vacuoles, banded nuclei, relaxed chromatin and abundant cytoplasm. Fig. 1C (bottom panel) demonstrates that the number of YY1-positive cells was significantly increased in patients with sepsis compared with healthy controls $(\mathrm{P}=0.011)$. The potential correlation of Fas and YY1 expression with levels of apoptosis was also analyzed. Fig. 1D demonstrates that there was a positive correlation between Fas expression and the level of apoptosis $(r=0.5013$, $\mathrm{P}=0.020$ ). These results demonstrate that apoptosis and the expression of Fas are increased in patients with sepsis, and that the levels of apoptosis are positively correlated with the expression of Fas. This indicates that the apoptosis induced in patients with sepsis may be mediated by Fas expression. By contrast, an inverse correlation was detected between YY1 expression and apoptosis levels ( $\mathrm{r}=-0.4988, \mathrm{P}=0.049$; Fig. 1E). Together, these results suggest that YY1 may inhibit apoptosis via regulation of Fas expression. Additionally, the percentage of FasL-positive cells was significantly higher in patients with sepsis compared with the healthy controls ( $\mathrm{P}=0.033$; Fig. 1F).
Apoptosis of PBMCs has a direct association with sepsis. To define the potential role of apoptosis in the pathogenesis of sepsis, comparative analyses of apoptosis and the different clinical stages of the patients was performed. As presented in Fig. 2A, comparing apoptosis between patients with sepsis only (group 1) and those with sepsis and MOD (group 2) demonstrated that patients with sepsis exhibited increased levels of apoptosis $(\mathrm{P}=0.001)$ compared with patients with MOD. In addition, the association between the outcome of the patient and apoptosis was analyzed (Fig. 2B). The results demonstrated that there is an inverse association between the patient outcome and apoptosis, as the level of apoptosis was higher in patients that did not survive compared with those that did survive $(\mathrm{P}=0.034)$.

Positive correlation between Fas expression and PELOD index in patients with sepsis. The PELOD index is useful in establishing prognosis during sepsis. In the current study, the correlation of the PELOD index with apoptosis was analyzed. Interestingly, a direct positive correlation between the level of apoptosis and the PELOD index was detected $(\mathrm{P}=0.01, \mathrm{r}=0.413$; Fig. 3A). The study also analyzed the correlation of Fas and YY1 expression with the PELOD index. Fig. 3B demonstrates that there was a positive correlation between Fas expression and PELOD ( $\mathrm{P}=0.026, \mathrm{r}=0.24)$. By contrast, the expression of YY1 and the PELOD index were inversely correlated $(\mathrm{P}=0.21$, $\mathrm{r}=-0.29$; Fig. 3C). In addition, the association of the expression of Fas and YY1, and patient outcome was analyzed. The results demonstrated that there was no correlation between the expression of Fas and a fatal outcome. Similarly, no correlation between YY1 expression and patient outcome was detected (data not shown).

YY1 expression as a biomarker of outcome in patients with sepsis. The present study analyzed whether YY1 expression and apoptosis levels may predict the clinical outcome of patients with sepsis. The cohort was analyzed for high/low YY1 expression and apoptosis. High YY1 expression was defined as $10 \%$ of cells positive for YY1 and apoptosis was considered high with $>30 \%$ of cells positive for apoptosis. 

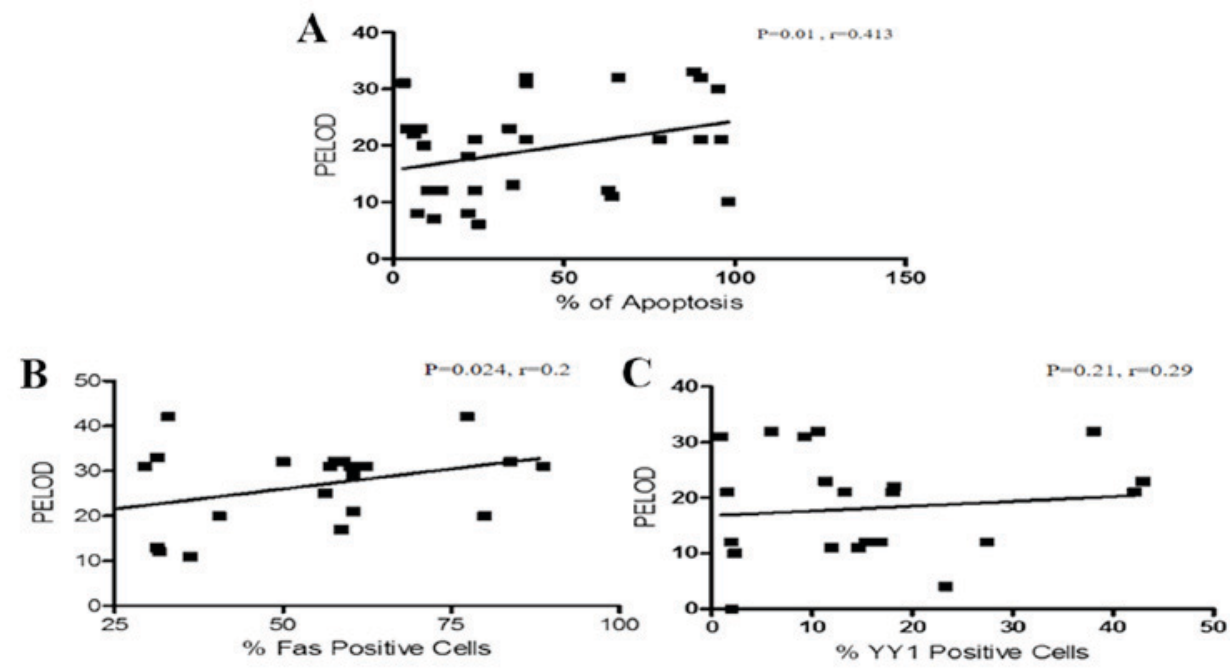

Figure 3. Correlation analysis between the PELOD index and apoptosis, Fas expression and YY1 expression. (A) Correlation analysis between apoptosis levels and PELOD index in PBMCs from pediatric patients with sepsis. There was a positive correlation between the PELOD index and apoptosis. (Spearman test, $\mathrm{P}=0.01, \mathrm{r}=0.413$ ). (B) Correlation analysis of Fas expression and PELOD index in PBMCs derived from pediatric patients with sepsis. There was a positive correlation between the PELOD index and Fas expression (Spearman test, $\mathrm{P}=0.024, \mathrm{r}=0.2$ ). (C) Correlation analysis between YY1 expression and PELOD Index in PBMCs derived from pediatric patients with sepsis. There is no significant correlation between YY1 expression and the PELOD index. (Spearman test, $\mathrm{P}=0.21$, r= 0.29). PELOD, pediatric logistic organ dysfunction; PBMC, peripheral blood mononuclear cell; Fas, Fas cell surface death receptor; YY1, YY1 transcription factor.
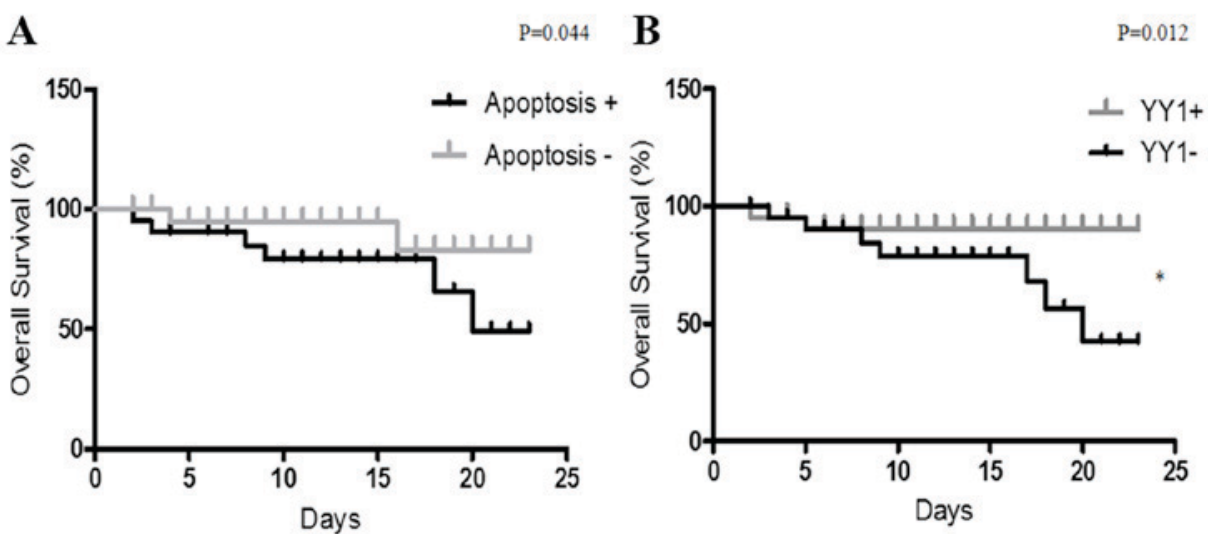

Figure 4. Survival curves for pediatric patients with sepsis with different levels of apoptosis or YY1 expression. (A) Percentage overall survival curve of patients with high/low apoptosis in peripheral blood mononuclear cells analyzed by terminal deoxynucleotidyl transferase dUTP nick-end labeling. High, 48\% survival ( $\mathrm{n}=18)$; low, 83\% survival ( $\mathrm{n}=12)$. $\mathrm{P}=0.044$. (B) Percentage survival curve for pediatric patients with sepsis with high/low $\mathrm{YY} 1 \mathrm{expression}$. High, 90\% survival $(\mathrm{n}=16)$; low, $42 \%$ survival $(\mathrm{n}=14)$. $\mathrm{P}=0.012$. $\mathrm{YY} 1$, $\mathrm{YY} 1$ transcription factor.

As presented in Fig. 4, the clinical outcomes of patients with sepsis were compared with YY1 expression and apoptosis by Kaplan-Meier analysis. The follow-up (23 days) overall survival scores were 83 and $49 \%$ for patients with low apoptosis and high apoptosis, respectively (Fig. 4A). Additionally, the follow-up overall survival scores were 90 and $42 \%$ for patients with high YY1 and low YY1 patients, respectively $(\mathrm{P}=0.012$ Fig. 4B). The results demonstrated that high apoptosis and low YY1 expression are indicators of poor overall survival in patients with sepsis. In addition, survival was compared with PELOD index. Fig. 5 revealed that a low PELOD index was associated with increased survival $(\mathrm{P}=0.021)$.

\section{Discussion}

Apoptosis is a cell self-destruction mechanism that has an important role in tissue homeostasis, embryonic development and control of the immune response $(22,23)$. However, when apoptosis is activated at an inappropriate time, it can cause the death of important cells in the immune system $(24,25)$. Lymphocyte apoptosis is important for the function of the immune response (26), as it is involved in the positive and negative selection of $\mathrm{B}$ and $\mathrm{T}$ cells (27). Apoptosis also regulates the intensity and duration of the immune response, as activated lymphocytes undergo apoptosis following an immune response to limit inflammation (28). However, when 


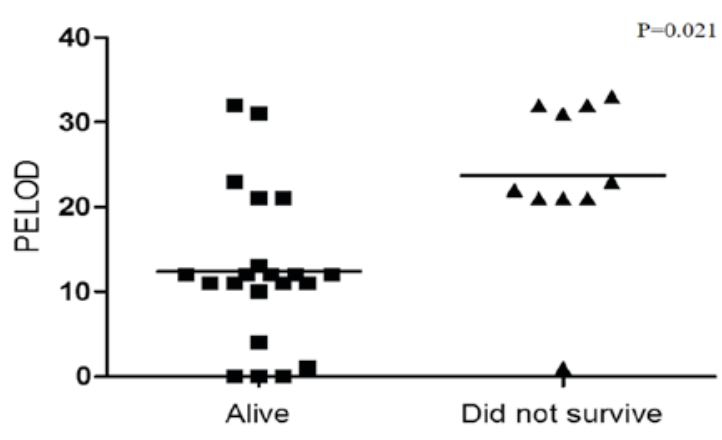

Figure 5. Frequency of PELOD index in PBMCs from pediatric patients with sepsis. PELOD index was compared with outcome of patients with sepsis. (Student's t-test, $\mathrm{P}=0.021$, alive vs. did not survive). PELOD, pediatric logistic organ dysfunction; PBMC, peripheral blood mononuclear cell.

apoptosis malfunctions, the results can be serious and can be damaging for the organism.

Apoptosis is also an important mechanism in the pathogenesis of MOD in sepsis. It has been previously reported that apoptosis of neutrophils and lymphocytes is an early event during sepsis and MOD. The half-life of a circulating neutrophil is $6-20 \mathrm{~h}$, and they can survive $>48 \mathrm{~h}$ at sites of inflammation (29). It is known that there are high numbers of apoptotic lymphocytes in specific organs or in the periphery of patients with sepsis that subsequently developed MOD. The majority of children that die from severe sepsis and MOD do not fully eradicate the infection $(30,31)$. This indicates that the cellular components of the immune response may be compromised in patients with MOD (32). A previous study has demonstrated that lymphocyte apoptosis is associated with the development of severe sepsis and MOD, as in an experimental model of sepsis the inhibition of apoptosis reduced bacteremia and increased survival (33). Lymphocyte apoptosis may be altered by steroids, cytokines, including TNF- $\alpha$, IL-1 and IL-6, oxygen free radicals, nitric oxide and T cells expressing FasL (34). There is an association between apoptosis in peripheral cells, or other tissue-specific cell types, with the severity of sepsis and the development of MOD, and this apoptosis is mediated by Fas/FasL expression, however, the potential mechanisms that regulate the expression of Fas/FasL are not fully established (35).

The current study analyzed apoptosis levels, and the expression of Fas and YY1, in PBMCs from children with sepsis, and investigated associations with clinical data (including one of the most serious complications of sepsis, MOD). To analyze apoptosis levels, a control group of healthy individuals were compared with the patients with sepsis. The results demonstrated a significant increase in PBMC apoptosis levels in patients with sepsis compared with the controls. In addition to TUNEL staining, morphological changes were observed in the cells from patients with sepsis, including changes to the cytoplasm, vacuoles and nuclei. A previous study has demonstrated the association between apoptosis mediated by Fas/FasL expression, and the severity of sepsis and MOD (35), however, the potential mechanisms of Fas regulation in this context remain unknown. Previous studies by our group and others have demonstrated that the transcription factor YY1 negatively regulates Fas expression, and inhibition of this transcription factor leads to increased expression of Fas, which results in an increased susceptibility to apoptosis in tumor cells $(36,37)$. The results of the present study demonstrated that there was significant overexpression of Fas and FasL in PBMCs from patients with sepsis compared with healthy controls (Fig. 1B and F). A previous study has reported that Fas and FasL expression is tissue specific (35). However, the current study is the first, to the best of our knowledge, to analyze Fas expression in PBMCs from children with sepsis. Additionally, tissue-specific apoptosis was previously demonstrated to be correlated with Fas expression in PBMCs (4). Similarly, the correlation analysis of the present study demonstrated that PBMC apoptosis was positively correlated with Fas expression. The present study has particular importance as, to the best of our knowledge, it is the first to investigate the expression of YY1 in PBMCs from patients with sepsis, and to investigate its correlation with Fas expression and apoptosis. The results highlighted that YY1 was predominantly expressed in the nuclei of PBMCs (Fig. 1C). The increased expression of YY1 detected was modest and other studies have reported contradictory results (38), demonstrating that activated T cells have high YY1 expression. Sepsis is induced by the activation of T lymphocytes following infection, and high levels of YY1 are expressed in PBMCs from patients with sepsis. However, sepsis is initially a pro-inflammatory process that progresses into an anti-inflammatory state, which is predominantly mediated by apoptosis. However, it is important to consider that all of these stages may occur simultaneously (39). We hypothesize that this phenomenon induces downregulation of YY1 and contributes to the induction of apoptosis in PBMCs cells, leading to an immunosuppressive state that triggers severe sepsis, septic shock and MOD. The current study demonstrated that apoptosis was inversely correlated with YY1 expression (Fig. 1E). These results indicated that YY1 expression may have an anti-apoptotic effect, partially by downregulation of the expression pro-apoptotic proteins, including Fas. However, the results did not indicate that there was an inverse correlation between Fas and YY1 expression (data not shown). This was potentially due to the low levels of YY1 expression in the PBMCs from the patients with sepsis and, it was therefore difficult to establish a clear negative correlation with the levels of Fas expression.

Previous studies have demonstrated that YY1 expression in hematological malignances, including lymphoma and leukemia, is associated with patient outcome $(40,41)$, suggesting that YY1 expression has an important role in these diseases and, consistent with the results of the present study, can potentially be used as a prognostic biomarker to predict outcome in pediatric patients.

The results of the present study demonstrated that pediatric patients with sepsis have higher PBMC apoptosis compared with septic patients that developed MOD (Fig. 2A). These findings are not consistent with a previous report, which observed that apoptosis was positively correlated with the development of MOD (42). However, the results of the present study indicated that PBMCs exhibited high apoptosis in the early stages of sepsis, which diminished following MOD development. These results were corroborated by analyzing the association between apoptosis levels and patient outcome (Fig. 2B). The results demonstrated that patients with high apoptosis levels 
have a poorer survival outcome compared with patients with low apoptosis levels. This indicated that although apoptosis is involved in immunosuppression of these patients, it may also have a compensatory role and, therefore, prevent progression to MOD and promote survival.

As discussed, MOD is the most common cause of death in the pediatric ICU. Determination of the severity of this syndrome may be useful for predicting disease progression. The PELOD index is currently used to assess the severity of MOD in children with sepsis (43). The current study analyzed the association of the PELOD index with the outcome of patients, and demonstrated that mortality is clearly increased in patients with a greater PELOD index (up to 20), which is consistent with previous reports $(43,44)$ (Fig. 5). As expected, there was a direct positive correlation between the PELOD index and apoptosis levels in PBMCs (Fig. 3A). These results indicate that apoptosis is not a protective factor in these patients. Apoptosis may be useful in clinical evaluation as a potential prognostic marker. To the best of our knowledge, the current study is the first to demonstrate a positive correlation between the PELOD index and apoptosis in PBMCs from pediatric patients with sepsis. A positive correlation between the PELOD index and Fas expression (Fig. 3B), and an inverse correlation between the PELOD index and YY1 expression (Fig. 3C) were also observed. These results indicate that Fas may mediate apoptosis induction and is correlated with a high PELOD index, and YY1 has a negative role in the regulation of Fas expression and reduces apoptosis to improve the prognosis of patients with sepsis. Finally, the results demonstrated that apoptosis is a prognostic factor for poor outcomes in pediatric patients with sepsis (Fig. 4A).

To the best of our knowledge, the present study is the first report to investigate YY1 expression in PBMC from patients with sepsis. We hypothesize that YY1 expression and apoptosis levels may be used as markers, in combination with others, to predict the outcome of patients with sepsis.

Further studies are required to determine the mechanisms that underlie the association of YY1 expression with clinical outcome. In addition, determining cytokine secretion, and its association with the expression of YY1 may be important, as will determining the prognostic index of YY1 and the anti-apoptotic Bcl-2 protein in pediatric patients with sepsis.

\section{Acknowledgements}

The present study was partially supported by the Health Research Fundings (grant MIV FIS/IMSS/PROT/G09/759) from the IMSS. The authors acknowledge the participation of Brenda Viridiana González Revilla, Vanessa Retana Mayen, Miriam Hernández Atenógenes and Juan Carlos Vázquez Islas, (Immunology \& Infections Diseases Medical Research Unit, Medical Center La Raza IMSS), for their participation in the realization of this work and to Mario Morales-Martinez (UIMEO National Medical Center Siglo XXI, IMSS) in the preparation of the manuscript.

\section{References}

1. Hotchkiss RS and Nicholson DW: Apoptosis and caspases regulate death and inflammation in sepsis. Nat Rev Immunol 6: 813-822, 2006
2. Rayo AC, Hernández GL, Huerta GL, Ortiz AV, Reyes RM and Gómez RG: Mecanismos patogénicos en sepsis y choque séptico. Med Interna Mex 24: 38-42, 2008

3. Carrillo-Esper R, Carrillo-Cordova JR and Carrillo-Cordova LD: Epidemiological study of sepsis in Mexican intensive care units. Cir Cir 77: 279-385, 2009 (In Spanish).

4. Hotchkiss RS, Swanson PE, Freeman BD, Tinsley KW, Cobb JP, Matuschak GM, Buchman TG and Karl IE: Apoptotic cell death in patients with sepsis, shock, and multiple organ dysfunction. Crit Care Med 27: 1230-1251, 1999.

5. Hotchkiss RS, Swanson PE, Cobb JP, Jacobson A, Buchman TG and Karl IE: Apoptosis in lymphoid and parenchymal cells during sepsis: Findings in normal and T- and B-cell-deficient mice. Crit Care Med 25: 1298-1307, 1997.

6. Hotchkiss RS, Swanson PE, Knudson CM, Chang KC, Cobb JP, Osborne DF, Zollner KM, Buchman TG, Korsmeyer SJ and Karl IE: Overexpression of Bcl-2 in transgenic mice decreases apoptosis and improves survival in sepsis. J Immunol 162: 4148-4156, 1999.

7. Chung CS, Song GY, Moldawer LL, Chaudry IH and Ayala A: Neither fas ligand nor endotoxin is responsible for inducible peritoneal phagocyte apoptosis during sepsis/peritonitis. J Surg Res 91: 147-153, 2000.

8. Hotchkiss RS, Osmon SB, Chang KC, Wagner TH, Coopersmith CM and Karl IE: Accelerated lymphocyte death in sepsis occurs by both the death receptor and mitochondrial pathways. J Immunol 174: 5110-5118, 2005.

9. Niu J, Azfer A and Kolattukudy PE: Protection against lipopolysaccharide-induced myocardial dysfunction in mice by cardiac-specific expression of soluble Fas. J Mol Cell Cardiol 44: 160-169, 2008.

10. Ren Y, Xie Y, Jiang G, Fan J, Yeung J, Li W, Tam PK and Savill J: Apoptotic cells protect mice against lipopolysaccharide-induced shock. J Immunol 180: 4978-4985, 2008.

11. Gautier EL, Huby T, Saint-Charles F, Ouzilleau B, Chapman MJ and Lesnik P: Enhanced dendritic cell survival attenuates lipopolysaccharide-induced immunosuppression and increases resistance to lethal endotoxic shock. J Immunol 180: 6941-6946, 2008.

12. Unsinger J, Herndon JM, Davis CG, Muenzer JT, Hotchkiss RS and Ferguson TA: The role of TCR engagement and activation-induced cell death in sepsis-induced $\mathrm{T}$ cell apoptosis. J Immunol 177: 7968-7973, 2006.

13. Ayala A, Urbanich MA, Herdon CD and Chaudry IH: Is sepsis-induced apoptosis associated with macrophage dysfunction? J Trauma 40: 568-574, 1996.

14. Sharshar T, Gray F, Poron F, Raphael JC, Gajdos P and Annane D: Multifocal necrotizing leukoencephalopathy in septic shock. Crit Care Med 30: 2371-2375, 2002.

15. Gordon S, Akopyan G, Garban H and Bonavida B: Transcription factor YY1: Structure, function, and therapeutic implications in cancer biology. Oncogene 25: 1125-1142, 2006.

16. Huerta-Yepez S, Vega M, Garban H and Bonavida B: Involvement of the TNF-alpha autocrine-paracrine loop, via NF-kappaB and YY1, in the regulation of tumor cell resistance to Fas-induced apoptosis. Clin Immunol 120: 297-309, 2006.

17. Garbán HJ and Bonavida B: Nitric oxide inhibits the transcription repressor Yin-Yang 1 binding activity at the silencer region of the Fas promoter: A pivotal role for nitric oxide in the up-regulation of Fas gene expression in human tumor cells. J Immunol 167: 75-81, 2001.

18. De Freitas I, Fernández-Somoza M, Essenfeld-Sekler E and Cardier JE: Serum levels of the apoptosis-associated molecules, tumor necrosis factor-alpha/tumor necrosis factor type-I receptor and Fas/FasL, in sepsis. Chest 125: 2238-2246, 2004.

19. Hotchkiss RS, Tinsley KW and Karl IE: Role of apoptotic cell death in sepsis. Scand J Infect Dis 35: 585-592, 2003.

20. Adrie C, Bachelet M, Vayssier-Taussat M, Russo-Marie F, Bouchaert I, Adib-Conquy M, Cavaillon JM, Pinsky MR, Dhainaut JF and Polla BS: Mitochondrial membrane potential and apoptosis peripheral blood monocytes in severe human sepsis. Am J Respir Crit Care Med 164: 389-395, 2001.

21. World Medical Association: World Medical Association Declaration of Helsinki: Ethical principles for medical research involving human subjects. JAMA 310: 2191-2194, 2013.

22. Holmström TH and Eriksson JE: Phosphorylation-based signaling in Fas receptor-mediated apoptosis. Crit Rev Immunol 20: 121-152, 2000.

23. Rathmell JC and Thompson CB: Pathways of apoptosis in lymphocyte development, homeostasis, and disease. Cell 109 (Suppl): S97-S107, 2002. 
24. Fadeel B and Orrenius S: Apoptosis: A basic biological phenomenon with wide-ranging implications in human disease. J Intern Med 258: 479-517, 2005.

25. Wyllie AH, Morris RG, Smith AL and Dunlop D: Chromatin cleavage in apoptosis: Association with condensed chromatin morphology and dependence on macromolecular synthesis. J Pathol 142: 67-77, 1984.

26. Porter BO and Malek TR: Prostaglandin E2 inhibits T cell activation-induced apoptosis and Fas-mediated cellular cytotoxicity by blockade of Fas-ligand induction. Eur J Immunol 29: 2360-2365, 1999.

27. McCarthy NJ, Smith CA and Williams GT: Apoptosis in the development of the immune system: Growth factors, clonal selection and bcl-2. Cancer Metastasis Rev 11: 157-178, 1992.

28. Newton K and Strasser A: Cell death control in lymphocytes. Adv Immunol 76: 179-226, 2000.

29. Toledano BJ, Bastien Y, Noya F and Mazer B: Characterization of B lymphocytes rescued from apoptosis by platelet-activating factor. Cell Immunol 191: 60-68, 1999.

30. Ashare A, Monick MM, Powers LS, Yarovinsky T and Hunninghake GW: Severe bacteremia results in a loss of hepatic bacterial clearance. Am J Respir Crit Care Med 173: 644-652, 2006

31. Stearns-Kurosawa DJ, Osuchowski MF, Valentine C, Kurosawa S and Remick DG: The pathogenesis of sepsis. Annu Rev Pathol 6: 19-48, 2011.

32. Hotchkiss RS, Tinsley KW, Swanson PE, Schmieg RE Jr, Hui JJ, Chang KC, Osborne DF, Freeman BD, Cobb JP, Buchman TG and Karl IE: Sepsis-induced apoptosis causes progressive profound depletion of B and CD4+ T lymphocytes in humans. J Immunol 166: 6952-6963, 2001.

33. Hotchkiss RS, Chang KC, Swanson PE, Tinsley KW, Hui JJ, Klender P, Xanthoudakis S, Roy S, Black C, Grimm E, et al: Caspase inhibitors improve survival in sepsis: A critical role of the lymphocyte. Nat Immunol 1: 496-501, 2000.

34. Roth E and Pircher H: IFN-gamma promotes Fas ligand- and perforin-mediated liver cell destruction by cytotoxic CD8 T cells. J Immunol 172: 1588-1594, 2004.

35. Doughty L, Clark RS, Kaplan SS, Sasser H and Carcillo J: sFas and $\mathrm{sFas}$ ligand and pediatric sepsis-induced multiple organ failure syndrome. Pediatr Res 52: 922-927, 2002.
36. Vega MI, Jazirehi AR, Huerta-Yepez S and Bonavida B: Rituximab-induced inhibition of YY1 and Bcl-xL expression in Ramos non-Hodgkin's lymphoma cell line via inhibition of NF-kappa B activity: Role of YY1 and Bcl-xL in Fas resistance and chemoresistance, respectively. J Immunol 175: 2174-2183, 2005.

37. Krippner-Heidenreich A, Walsemann G, Beyrouthy MJ, Speckgens S, Kraft R, Thole H, Talanian RV, Hurt MM and Lüscher B: Caspase-dependent regulation and subcellular redistribution of the transcriptional modulator YY1 during apoptosis. Mol Cell Biol 25: 3704-3714, 2005.

38. Guo J, Casolaro V, Seto E, Yang WM, Chang C, Seminario MC, Keen J and Georas SN: Yin-Yang 1 Activates Interleukin-4 Gene Expression in T Cells. J Biol Chem 276: 48871-48878, 2001.

39. Denlinger LC and Proctor RA: Potential risk factors for developing apoptosis during septic shock. Crit Care Med 28: 2133-2134, 2000.

40. Sakhinia E, Glennie C, Hoyland JA, Menasce LP, Brady G, Miller C, Radford JA and Byers RJ: Clinical quantitation of diagnostic and predictive gene expression levels in follicular and diffuse large B-cell lymphoma by RT-PCR gene expression profiling. Blood 109: 3922-3928, 2007.

41. Krammer PH: CD95(APO-1/Fas)-mediated apoptosis: Live and let die. Adv Immunol 71: 163-210, 1999

42. Hofer S, Brenner T, Bopp C, Steppan J, Lichtenstern C, Weitz J, Bruckner T, Martin E, Hoffmann U and Weigand MA: Cell death serum biomarkers are early predictors for survival in severe septic patients with hepatic dysfunction. Crit Care 13: R93, 2009.

43. Leteurtre S, Martinot A, Duhamel A, Proulx F, Grandbastien B, Cotting J, Gottesman R, Joffe A, Pfenninger J, Hubert P, et al: Validation of the paediatric logistic organ dysfunction (PELOD) score: Prospective, observational, multicentre study. Lancet 362: 192-197, 2003

44. Proulx F, Gauthier M, Nadeau D, Lacroix J and Farrell CA: Timing and predictors of death in pediatric patients with multiple organ system failure. Crit Care Med 22: 1025-1031, 1994. 